\title{
Estudio de Validación para el Contexto Ecuatoriano del Inventario APO Creado en Portugal
}

\author{
Validation for the Ecuadorian Context of the APO Inventory Created in Portugal
}

\author{
Valentina $\operatorname{Ramos}^{1}$, Stalyn Mejía ${ }^{1}$, Kléber Mejía ${ }^{1}$, Xavier L. Unda ${ }^{1}$ y Filomena Jordão ${ }^{2}$
}

\begin{abstract}
Resumen
El Inventario APO, creado para el contexto portugués, mide diferentes formas de congruencia entre valores personales y organizacionales. El presente estudio tiene por objetivo la validación del mismo Inventario APO, para Ecuador. Se utilizó la versión original de 19 preguntas y se aplicó a una muestra de 133 trabajadores de varias organizaciones. A través del AFE se redujeron a 18 ítems distribuidos en tres factores, explicando el $61,68 \%$ de la varianza con $\alpha_{\text {general }}=.758$. Los valores de $\alpha$ para cada factor fueron: $\alpha_{\text {ajuste }}=.857$, $\alpha_{\text {conflicto }}=.855$; y $\alpha_{\text {reconocimiento }}=.855$. Las diferencias en relación con el Inventario portugués estuvieron en el número de ítems final (Portugal=16 ítems, Ecuador=18 ítems) y al tipo de ítems removidos (Portugal=ítems 1, 5 y 19, Ecuador=ítem 2). Al igual que el estudio original los resultados muestran diferenciaciones conceptuales entre variables y resaltan la utilización de escalas independientes.
\end{abstract}

Palabras clave: ajuste persona-organización, conflicto de valores, reconocimiento de valores organizacionales, medición

\begin{abstract}
The APO Inventory was developed in Portugal, to measure different forms of person-organization value congruence. This study aims to validate the Inventory for the Ecuadorian context. For this, the Inventory with 19 questions was used and it was applied to a sample of 133 workers from various organizations. Through factor analysis, items were reduced and were divided into three factors, explaining the $61.68 \%$ of the variance. Values for general internal consistency were $\alpha_{\text {general }}=758$, and for each factor were: $\alpha_{\mathrm{fit}}=.857$, $\alpha_{\text {conflict }}=855$; and $\alpha_{\text {recognition }}=.855$. The differences regarding the same study in Portugal were associated with the number of final items (Portugal=16 items, Ecuador=18 items) and the type of items removed (Portugal=items 1, 5 and 19; Ecuador=item 2). Like the original study, results show the conceptual distinction between variables measured and highlight the use of separate scales.
\end{abstract}

Keywords: person-organization fit, value conflict, organizational values recognition, measurement

Este estudio se realizó bajo el proyecto financiado por la Escuela Politécnica Nacional con Referencia: PII-DESODEH-0032015

${ }^{1}$ Facultad de Ciencias Administrativas, Escuela Politécnica Nacional, Diego Ladrón de Guevara, EC170143, Quito, Ecuador, Tel.: +59322976300.

${ }^{2}$ Faculdade de Psicologia e de Ciências da Educação, Universidade do Porto, R. Alfredo Allen 535, Porto, Portugal. Tel.: +351 226079700 .

Correo: valentina.ramos@epn.edu.ec 


\section{Introducción}

Los valores dentro del contexto organizacional según Finegan (2000), son estándares evaluativos referentes al trabajo y al ambiente del trabajo por el cual los individuos disciernen qué es "correcto", o determinan la importancia de sus preferencias. Los individuos estarían probablemente más cómodos en un ambiente consistente con sus valores; si se les coloca en situaciones de desacuerdo con sus valores personales aparecerían efectos negativos en diferentes aspectos, tanto para el empleado como para la organización. Entonces los valores constituyen criterios que orientan las acciones de las personas y su conducta dentro de un espacio determinado debido a que proporcionan información sobre la forma cómo nos relacionamos con el mundo al tener integrados tanto un componente afectivo como conceptual o cognoscitivo (Rokeach, 1979).

Estudios previos como el de Ramos, Jordão y Morais (2012) miden el grado de congruencia existente entre los valores personales y organizacionales. Las variables asociadas a la congruencia fueron específicamente el ajuste o fit y el conflicto de valores, destacando especialmente que la falta de ajuste no implica la presencia de conflicto (Ramos, Jordão \& Morais, 2012).

\section{Propiedades Inventario APO}

El Inventario APO de Ramos, Jordão y Morais (2012) se desarrolló para medir simultáneamente la presencia del ajuste personaorganización, conflicto de valores y reconocimiento de valores en las organizaciones laborales. La validación de dicho instrumento se realizó para el contexto portugués. Originalmente se identificaron 19 ítems que fueron reducidos a 16 ítems después de la aplicación de la herramienta a una muestra de 102 trabajadores provenientes de empresas con diferente forma jurídica, sector, antigüedad y tamaño (Ramos, Jordão, \& Morais, 2012). Los ítems eliminados obedecen a dos razones: comprensión del mismo (ítem 1) y saturación en dos factores, con una diferencia inferior a 0.10 (ítems 5 y 19). El análisis factorial exploratorio (AFE) permitió verificar una distribución de los ítems en tres factores consistentes con el modelo teórico inicial. La herramienta obtuvo valores aceptables relacionados con la consistencia interna y la confianza $\left(\alpha_{\text {general }}>\right.$.6). Los valores de consistencia por factores fueron: $\alpha_{\text {ajuste }}=.909, \alpha_{\text {conflicto }}=.891 ; \mathrm{y}$ $\alpha_{\text {reconocimiento }}=.901$ (Ramos, Jordão, \& Morais, 2012).

\section{Resultados de la validación del Inventario APO para Portugal}

Los resultados obtenidos por Ramos, Jordão y Morais (2012) corroboraron las ideas propuestas por Talbot (2011) y Wheeler, Gallagher, Brouer, y Sablynski (2007), que proponen que la medición del ajuste entre los valores individuales y organizacionales es independiente de la medición del conflicto entre valores, lo cual representa la principal novedad de este estudio. De este modo, se obtuvo una herramienta para medir estos niveles de congruencia de valores, validada para el contexto portugués, constituyéndose en la primera herramienta de este tipo para ser aplicada en las organizaciones.

\section{El Inventario APO para Ecuador}

Ecuador actualmente cuenta con un contexto favorable para la realización de investigaciones relacionadas con la cultura organizacional. Existe una apertura para este tipo de investigaciones a partir de las políticas del Plan del Buen Vivir (Ecuador, 2013) y el Proyecto Nacional de Diálogo de Saberes (SENESCYT, 2015) apoyados por el Gobierno. Sin embargo, no existen herramientas que permitan estudiar la cultura, validada para este contexto.

Los valores han servido como punto de partida para la comprensión de la cultura en las organizaciones (Schein, 1996), permitiendo el desarrollo de modelos de gestión organizacional, así como constituyendo la base para el diagnóstico de la cultura en dependencia del contexto en donde se realiza el estudio (Dolan \& Altman, 2012; Ramos \& Jordão, 2012). Sin embargo, para comprender la influencia de los valores en la dinámica que se establece entre el trabajador y la organización dentro de la cultura organizacional se ha utilizado el concepto de congruencia de valores (Liedtka, 1989; Posner \& Schmidt, 1993). 
Estos elementos sirvieron como precedente para fundamentar, entre otras cuestiones, la necesidad de la validación del Inventario APO propuesto por Ramos, Jordão y Morais (2012) para el contexto organizacional ecuatoriano. Además, debe considerarse fundamentalmente la necesidad validar herramientas que se quieren aplicar en un contexto cuando fueron diseñadas para otro, así como lo demuestra el estudio de Silva, Oliveira, y Miguel (2015) y Major, Miranda, RodríguezGonzález y Relvas (2014).

En razón de lo expuesto, el objetivo fundamental de esta investigación radica en la obtención de un instrumento validado para Ecuador que permita medir el ajuste personaorganización, el conflicto de valores y el reconocimiento de valores como formas de congruencia entre valores organizacionales e individuales, con el propósito de ser aplicado desde un punto de vista académico y científico con vistas a comprender el comportamiento de la cultura organizacional en este contexto.

\section{Método}

\section{Participantes}

La selección de los participantes se basó en un muestreo no probabilístico. A partir de un grupo

de personas a las que se tenía acceso (como fue el caso de antiguos estudiantes de maestría) se avanzó a una estrategia de distribución del Inventario por "bola de nieve". De todos los que respondieron se incluyeron aquellos que estuvieran trabajando en el momento de completar el Inventario, siendo esta la única característica de control. La muestra se compuso por un total de 133 trabajadores: $59 \%$ hombres y $41 \%$ mujeres. Los participantes fueron trabajadores de varias empresas con diferente forma jurídica, tamaño y tiempo de creación de varias regiones de Ecuador con una tendencia mayoritaria de la provincia de Pichincha. La media de la edad de la muestra fue de 36 años $(\mathrm{DE}=8)$. El nivel de escolaridad predominante fue el de Maestría (62\%) seguido por Tercer Nivel (34\%). En cuanto al tiempo de trabajo, la muestra osciló entre menos de un año y 36 años en la misma organización. Los valores totales tanto de la muestra por trabajadores como de la caracterización de las organizaciones participantes aparecen en la Tabla 1.

Tabla 1. Descripción de la muestra de trabajadores y de organizaciones participantes

Descripción de la muestra de trabajadores participantes en el estudio
Descripción de la muestra de organizaciones participantes en el estudio

\begin{tabular}{|c|c|c|c|}
\hline Sexo & & ón Legal & \\
\hline Femenino & 54 & Públicas & 59 \\
\hline Masculino & 79 & Privadas & 73 \\
\hline & & Mixtas & 1 \\
\hline Edad & & edad & \\
\hline Entre 20 y 30 años & 37 & Entre 1 y 5 años & 50 \\
\hline Entre 31 y 40 años & 65 & Entre 6 y 10 años & 22 \\
\hline Entre 41 y 50 años & 22 & Más de 10 años & 61 \\
\hline Más de 51 años & 9 & & \\
\hline Años de trabajo en la organización & & & \\
\hline Menos de 4 años & 77 & Pequeña empresa & 10 \\
\hline Entre 4 y 10 años & 36 & Micro empresa & 18 \\
\hline Más de 10 años & 20 & Mediana empresa & 18 \\
\hline & & Gran empresa & 87 \\
\hline Escolaridad & & & \\
\hline Inferior a Tercer Nivel & 3 & & \\
\hline Tercer Nivel & 46 & & \\
\hline Maestría & 84 & & \\
\hline
\end{tabular}




\section{Instrumento}

El insumo fundamental para la versión final del Inventario APO para aplicar en Ecuador fue tomado del Material complementario 2. Inventario para la medición de la articulación entre la persona y la organización (versión traducida en lengua española) de Ramos, Jordão y Morais (2012). Las opciones de la escala de Likert de siete puntos se modificó. Las formulaciones originales "Estoy totalmente en desacuerdo - Concuerdo totalmente" pasaron a ser "Desacuerdo Total - Concuerdo totalmente", de acuerdo a las sugerencias de expertos dentro del contexto ecuatoriano, para garantizar una mejor comprensión de las alternativas de respuesta.

La versión del Inventario APO en lengua española fue presentada a un grupo de docentes con conocimiento del área de estudio que funcionaron como expertos. De esta manera se determinó el grado de comprensión de los ítems, así como la relación entre las preguntas y las dimensiones con las que se relacionaban. Fueron dadas sugerencias también en relación con orden de presentación de las preguntas. Para esto se consideró la versión original de 19 ítems del Inventario APO de Ramos, Jordão y Morais (2012).

Derivado de este proceso se identificaron inconsistencias en el cuestionario en la versión en lengua española que fueron corregidas como se señala a continuación:

- Los ítems 2 y 7 tuvieron la misma redacción en el momento de ser traducidos del idioma portugués al español. Para resolver esta situación, el ítem 7 fue reformulado respetando el original en portugués como "Los valores y la cultura de la organización a la que pertenezco son un reflejo de mis valores y mi cultura" reemplazando la formulación "Me identifico con los valores y la cultura de la organización a la que pertenezco".

- En el Inventario original en portugués el ítem 1 fue eliminado pues no se comprendía el sentido de la pregunta y saturaba un factor que no se correspondía con la base teórica de la herramienta. Como consecuencia se cambió el enunciado de "Sé cuáles son los valores y la cultura de la organización a la que pertenezco" por "Reconozco fácilmente los valores y la cultura de la organización a la que pertenezco".
- El ítem 11 hacía referencia al término "departamento" aludiendo al lugar de trabajo de las personas. Para facilitar la comprensión del término de acuerdo a su uso en el contexto ecuatoriano fue modificado del original "Las personas del departamento donde trabajo saben cuáles son los valores y la cultura de la organización" por la nueva forma "Las personas del área donde trabajo saben cuáles son los valores y la cultura de la organización";

- Los ítems 13 y 14 hacían referencia al término "valoriza" refiriéndose a la acción de valorar, término que no resultaba comprensible. Como consecuencia, el ítem 13 se modificó, adoptando la siguiente formulación "Los comportamientos que mi organización valoriza están en conflicto con mis valores personales" en reemplazo de "Los comportamientos que mi organización valora están en conflicto con mis valores personales". Asimismo, el ítem 14 se modificó, adoptando "Las cosas que yo valorizo en mi vida son semejantes a las cosas que la organización a la que pertenezco valoriza" en lugar de "Las cosas que yo valoro en mi vida son semejantes a las cosas que la organización a la que pertenezco valora".

Estos cambios permitieron el ajuste del instrumento final que fue aplicado a la muestra de trabajadores descrita (ver Anexo 1).

\section{Procedimiento}

El Inventario APO se colocó de forma digital utilizando para ello la herramienta de formularios de Google Docs 2014. La solicitud de participación en el estudio se envió a través del correo electrónico a una base de datos conformada por 2286 antiguos estudiantes de Maestría de la Escuela Politécnica Nacional, con la colaboración de la Facultad de Ciencias Administrativas.

En el Inventario se incluyó información relacionada con la obtención de datos sociodemográficos de la muestra. Para el análisis de los resultados, se tomaron en cuenta las respuestas de personas que estaban bajo algún tipo de régimen de trabajo en el momento de completar el Inventario. El uso de Google Docs permitió manejar la identidad del encuestado de manera confidencial, aspecto que fue indicado en la solicitud de colaboración en la que se adjuntaba el link del cuestionario. La herramienta estuvo 
Tabla 2. Análisis de componentes principales para el Inventario APO aplicado en Ecuador, distribuidos de acuerdo a una rotación Varimax con normalización Kaiser

\begin{tabular}{lccc}
\hline Ítems & $\begin{array}{c}\text { Ajuste entre valores } \\
\text { personales y } \\
\text { organizacionales }\end{array}$ & $\begin{array}{c}\text { Conflicto entre valores } \\
\text { personales y } \\
\text { organizacionales }\end{array}$ & $\begin{array}{c}\text { Reconocimiento de } \\
\text { valores } \\
\text { organizacionales }\end{array}$ \\
\hline Ítem 3 & .803 & & \\
Ítem 11 & .768 & & \\
Ítem 8 & .751 & & \\
Ítem 1 & .713 & & \\
Ítem 5 & .657 & .854 & \\
Ítem 12 & .632 & .830 & \\
Ítem 13 & & .783 & \\
Ítem 4 & & .749 & \\
Ítem 6 & & .656 & .711 \\
Ítem 18 & & .636 & .701 \\
Ítem 10 & & & .686 \\
Ítem 15 & & & .683 \\
Ítem 17 & & & .667 \\
Ítem 19 & & & .467 \\
Ítem 7 & & & \\
Ítem 16 & & & \\
Ítem 14 & & & \\
Ítem 9 & & & \\
Ítem 2 & & & \\
\hline
\end{tabular}

Nota: Para una mejor comprensión de los resultados eliminamos las interacciones inferiores a .40. El método de extracción utilizado fue el análisis de componentes principales. La rotación fue convergente en 5 interacciones. *indica el ítem removido debido a una saturación en dos factores con una diferencia inferior a .10.

disponible durante un período de tres meses aproximadamente.

\section{Análisis de los datos}

Para la reducción de ítems a factores se utilizó un análisis de componentes principales. Para el uso de este método se utilizaron los valores de los indicadores del test de Kaiser-Meyer-Olkin (KMO) y el test de Bartlett. Se consideró eliminar ítems que presentaron una saturación en más de un factor con una diferencia inferior a 0.10 de acuerdo con Stevens (1986) y con valores de comunalidad inferiores a 0.60 (Hinkin, 1998). Para la estimación de la confiabilidad del cuestionario y de cada factor obtenido se utilizó el coeficiente alfa de Cronbach (1951). Este conjunto de elementos constituyeron la base para el Análisis Factorial Exploratorio (AFE). Los cálculos se realizaron utilizando el programa estadístico SPSS 18.

\section{Resultados}

La validación del instrumento se realizó siguiendo la metodología propuesta por Hinkin (1998) y que fue seguida en la validación del cuestionario inicial de Ramos, Jordão y Morais (2012). Para ello se realizó un Análisis Factorial Exploratorio (AFE) que permitió reducir los ítems a factores, corroborando la saturación de los ítems para cada factor y la comprobación de la presencia de las dimensiones deseadas en esta versión del Inventario.

Después de aplicada la herramienta a la muestra seleccionada, se obtuvo una distribución de aproximadamente seis ítems por factor (ratio 6:1). Esta relación resultó superior al mínimo de 4 mencionado por Hinkin (1998) para probar la homogeneidad de ítems dentro de cada factor. Esta distribución permitió dar continuidad al 
Tabla 3. Análisis de componentes principales para el Inventario APO aplicado en Ecuador después eliminado el ítem 2, distribuidos de acuerdo a una rotación Varimax con normalización Kaiser

$\begin{array}{cccc}\text { Ítems } & \begin{array}{c}\text { Ajuste entre valores } \\ \text { personales y } \\ \text { organizacionales }\end{array} & \begin{array}{c}\text { Conflicto entre valores } \\ \text { personales y } \\ \text { organizacionales }\end{array} & \begin{array}{c}\text { Reconocimiento de } \\ \text { valores organizacionales }\end{array} \\ & & \end{array}$

\begin{tabular}{ll}
\hline Ítem 3 & .801 \\
Ítem 11 & .774 \\
Ítem 8 & .755 \\
Ítem 1 & .704 \\
Ítem 5 & .664 \\
Ítem 12 & .629
\end{tabular}

Ítem 13

Ítem $4 \quad .833$

Ítem $6 \quad .786$

Ítem $18 \quad .749$

Ítem $10 \quad .658$

Ítem $15 \quad .639$

Ítem $17 \quad 0.714$

Ítem $19+702$

Ítem $7 \quad$.688

Ítem $16 \quad .679$

Ítem $14 \quad .668$

Ítem $9 \quad .660$

Nota: Para una mejor comprensión de los resultados eliminamos las interacciones inferiores a 0.40 . El método de extracción utilizado fue el análisis de componentes principales. La rotación fue convergente en 5 interacciones.

estudio en función de encontrar evidencia de validez de la herramienta.

\section{Validez del Inventario APO en Ecuador en comparación con la versión portuguesa}

El análisis de componentes principales se realizó con rotación Varimax. El valor del índice de Kaiser-Meyer-Olkin $(\mathrm{KMO}=.850)$ resultó cercano al del estudio original que era de .898 . Los resultados del test de esfericidad de Bartlett $\left(x^{2}=452,96 ; p=.000\right)$ también fueron cercanos a los obtenidos en el estudio previo en el contexto portugués $\left(x^{2}=1180,156 ; p=.000\right)$. Ambos valores son considerados excelentes de acuerdo con Hair, Anderson, Tatham y Black (2005).

El análisis de componentes principales para el Inventario aplicado en Ecuador indicó una distribución en tres componentes principales, explicando el 61,027 \% de la varianza. En el estudio original igualmente se identificaron tres componentes, sin embargo, la varianza explicada fue superior $(71.92 \%$, ). El porcentaje de varianza explicada en el análisis actual resulta aceptable de acuerdo con Hinkin (1998). La distribución de los ítems por factores encontrada en el estudio en Ecuador está representada en la Tabla 2.

Del total de ítems, se eliminó el ítem 2, correspondiente al enunciado "Me identifico con los valores y la cultura de la organización a la que pertenezco", debido a los parámetros de saturación propuestos por Stevens (1986). Con esta eliminación se redujeron los ítems de 19 a 18 . Después de volver a aplicar el análisis de componentes principales, se mantuvo la distribución en tres componentes principales. La 
Tabla 4. Valores para la consistencia interna del Inventario APO para Portugal y para Ecuador

\begin{tabular}{lcccc}
\hline \multirow{2}{*}{$\begin{array}{l}\text { Escala General y distribución en } \\
\text { Factores }\end{array}$} & \multicolumn{4}{c}{ Portugal } \\
\cline { 2 - 5 } & $\begin{array}{c}\text { Número de } \\
\text { ítems }\end{array}$ & $\alpha$ & $\begin{array}{c}\text { Número de } \\
\text { ítems }\end{array}$ & $\alpha$ \\
\hline $\begin{array}{l}\text { Escala general } \\
\quad \begin{array}{l}\text { Ajuste entre valores personales } \\
\text { y organizacionales }\end{array}\end{array}$ & 16 & .608 & 18 & .758 \\
$\begin{array}{l}\text { Conflicto entre valores } \\
\text { personales y organizacionales }\end{array}$ & 6 & .909 & 6 & .857 \\
$\begin{array}{l}\text { Reconocimiento de valores } \\
\text { organizacionales }\end{array}$ & 6 & .891 & 6 & .855 \\
\hline
\end{tabular}

varianza explicada por estos tres componentes aumentó a $61,68 \%$ y los indicadores continuaron siendo satisfactorios para la reducción por factores $\left(\mathrm{KMO}=0.871 ; \quad x^{2}=1318,44 ; \quad p=.000\right)$. Los resultados del segundo análisis se muestran en la Tabla 3.

\section{Confiabilidad del Inventario APO en Ecuador en comparación con la versión portuguesa}

De acuerdo con Hinkin (1998), la confiabilidad es la exactitud o precisión de un instrumento de medición. Para estimar la confiabilidad de este instrumento calculamos el coeficiente alfa de Cronbach (1951). Los resultados comparativos de los valores de alfa para el Inventario en general y para cada dimensión, así como su comparación con la herramienta original se encuentran en la Tabla 4.

Tanto el Inventario APO validado en Portugal como el validado en Ecuador mantuvieron los mismos ítems distribuidos en las dimensiones ajuste y conflicto. El Inventario APO en Ecuador ganó dos ítems más para la dimensión reconocimiento de valores.

\section{Conclusiones}

La presente investigación permitió la validación del Inventario APO en el contexto ecuatoriano. De acuerdo al análisis realizado se obtuvieron tres factores medibles, confirmando resultados anteriores en su validación en Portugal (ajuste persona y organización, conflicto entre valores personales y valores organizacionales y reconocimiento de los valores organizacionales). Esta comparación permite que se establezca una congruencia entre la teoría y la práctica asociada a la medición de estas dimensiones.

Otros elementos que resultaron de interés fueron la obtención de valores semejantes de los coeficientes relacionados con la aplicación del análisis de componentes principales. Sin embargo, el Inventario APO para Portugal tuvo un mejor índice de varianza explicada para la distribución en tres componentes. Para la versión final en Ecuador se eliminó el ítem 2 pues saturaba dos factores. Aun así, esta cantidad de ítems eliminados fue inferior a la realizada en Portugal donde se eliminaron tres ítems de los 19 iniciales. De esta forma, el Inventario APO para Ecuador está conformado por 18 ítems, dos adicionales en la dimensión "reconocimiento de valores" respecto de la versión portuguesa.

Por otra parte cabe señalar que el Inventario APO para Ecuador obtuvo mejor índice de alfa general en comparación con el estudio efectuado en Portugal. Sin embargo, los valores de alfa por dimensiones fueron ligeramente superiores en el Inventario APO portugués respecto del ecuatoriano.

\section{Limitaciones y pistas para futuras investigaciones}

Tal como se indicara en el estudio realizado en Portugal por Ramos, Jordão y Morais (2012), se necesitaría la realización de un Análisis Factorial Confirmatorio para comprobar la interdependencia de las variables que aparecen en la distribución de factores. Por otra parte, este tipo 
de estudio da lugar a comparaciones entre los contextos donde la herramienta fue validada para posteriores comparaciones y estudios transculturales. Asimismo, sirve de preámbulo para que este Inventario comience a ser utilizado en las investigaciones sobre valores y cultura en Ecuador, lo cual constituye su principal aporte dentro del contexto donde se validó.

\section{Referencias}

Boxx, W. R., \& Odom, R. Y. (1991). Organizational values and value congruency and their impact on satisfaction, commitment, and cohesion. Public Personnel Management, 20(2), 195-206.

Cable, D. M., \& DeRue, D. S. (2002). The convergent and discriminant validity of subjective fit perceptions. Journal of Applied Psychology, 87(5), 875-884.

Caldwell, S. D., Herold, D. M., \& Fedor, D. B. (2004). Toward and understanding of the relationships among organizational change, individual differences, and changes in personenvironment fit: A cross-level study. Journal of Applied Psychology, 89(5), 868882.

Chatman, J. A. (1989). Matching people and organizations: Selection and socialization in public accounting firms. Academy of Management Best Papers Proceedings, 199203.

Cronbach, L. J. (1951). Coefficient alpha and the internal structure of tests. Psychometrika, 16(3), 297-334.

Davis, V. A. (2006). Relationships among subjective workplace fit perceptions, job satisfaction, organizational citizenship behavior, organizational commitment, and turnover intentions. Unpublished doctoral dissertation. Alliant International University, San Diego CA.

Dolan, S. L., \& Altman, Y. (2012). Managing by values: The leadership spirituality connection. People \& Strategy, 35(4), 20-26.

Ecuador, G. N. de la R. del. (2013). Buen vivir plan nacional. Quito: SENPLADES.
Edwards, J. R., \& Cable, D. M. (2009). The value of value congruence. Journal of Applied Psychology, 94(3), 654-677.

Finegan, J. (2000). The impact of person and organizacional values on organizacional commitment. Journal of Occupational an Organizational Psychology, 227.

Hair, J., Anderson, R., Tatham, R., \& Black, W. (2005). Análise multivariada de dados. Porto Alegre: Bookman.

Hinkin, T. R. (1998). A brief tutorial on the development of measures for use in survey questionnaires. Organizational Research Method, 112-113.

Kristof, A. L. (1996). Person-organization fit: an integrative review of its conceptualizations, measurement, and implications. Personnel Psychology, 49(1), 1-49.

Liedtka, J. M. (1989). Value congruence: The interplay of individual and organizational value systems. Journal of Business Ethics, 8(10), 805-815.

Major, S., Miranda, C., Rodríguez-González, M., \& Relvas, A. P. (2014). Adaptação portuguesa do Differentiation of Self Inventory-Revised (DSI-R): Um estudo exploratório. Portuguese adaptation of the Differentiation of Self Inventory-Revised (DSI-R): An exploratory study. Revista Iberoamericana de Diagnóstico y Evaluación - e Avaliação Psicológica, 1(37), 99-121.

Martin, J. Y. (1983). Organizational culture and counterculture: An uneasy symbiosis. Organizational Dynamics, 12, 52-64.

Martorell, C., \& Gómez, O. (2010). Enfoque de la evaluación psicológica de la Revista Iberoamericana de Diagnóstico y Evaluación - e Avaliação Psicológica (RIDEP). Revista Iberoamericana de Diagnóstico y Evaluación - e Avaliação Psicológica, 30(2), 35-46.

Medina, F. J., Munduate, L., Dorado, M. A., Martínez, I., \& Guerra, J. M. (2005). Types of intragroup conflict and affective reactions. Journal of Managerial Psychology, 20(3-4).

Meglino, B. M. (1998). Individual values in organizations: Concepts, controversies, and research. Journal of Management, 24(3), 351389. 
Posner, B. (2010). Another look at the impact of personal and organizational values congruency. Journal of Business Ethics, 97(4), 535-541.

Posner, B. Z., \& Schmidt, W. H. (1993). Values congruence and differences between the interplay of personal and organizational value systems. Journal of Business Ethics, 12, 341347. doi:10.1007/BF00882023

Ramos, V., \& Jordão, F. (2012). The relationship between cultural and non-cultural elements as a way to describe organizational context. Revista Psicologia.com, 16(7), 1-25. Retrieved from http://hdl.handle.net/10401/54 59

Ramos, V., Jordão, F., \& Morais, T. (2012). Desarrollo y validación del Inventario para Medir la Articulación entre la Persona y la Organización (Inventario APO). Evaluar, 6082.

Rokeach, M. (1979). Understanding human values: Individual and societal. New York: The Free Press.

Schein, E. H. (1996). Culture: The missing concept in organization studies. Administrative Science Quarterly, 41(2), 229240.

SENESCYT. (2015). Proyecto Nacional de Diálogo de Saberes. Retrieved August 18, 2015, from http://www.educacionsuperior.gob .ec/proyecto-nacional-de-dialogo-de-saberes/

Silva, J. M. T. da, Oliveira, A. L., \& Miguel, J. P. (2015). Adaptação e Validação Transcultural de uma Medida de Atitudes acerca da Estatística Cross-cultural. Revista Iberoamericana de Diagnóstico y Evaluación - e Avaliação Psicológica, 1(39), 102-112.

Silverthorne, C. (2004). The impact of organizational culture and personorganization fit on organizational commitment and job satisfaction in Taiwan. Leadership \& Organization Development Journal, 25(7), 592-599.

Stevens, J. (1986). Applied multivariate statistics for the social sciences. New Jersey: Lawrence Erbaum.

Talbot, D. y. (2011). Similarities \& differences between fit \& misfit. Trabajo presentado en la $15^{\mathrm{a}}$ Conferencia de la Asociación Europea de
Psicología Organizacional y del Trabajo, Maastricht.

Vandenberghe, C., \& Peiro, J. M. (1999). Organizational and individual values: Their main and combined effects on work attitudes and perceptions. European Journal of Work \& Organizational Psychology, 8(4), 569-581.

Vertel, A. d., Paternina, C. D., Riaño, H. E., \& Pereira, J. M. (2013). Cultura organizacional: evolución en la medición/Organizational culture: developments in measuring. Universidad Icesi, 4-6.

Wheeler, A. R., Gallagher, V. C., Brouer, R. L., \& Sablynski, C. J. (2007). When personorganization (mis)fit and (dis)satisfaction lead to turnover: The moderating role of perceived job mobility. Journal of Managerial Psychology, 22(2), 203-219.

Ximénez, M. C., \& Martín, R. S. (1998). El análisis de la conmensurabilidad en una medida de ajuste persona-ambiente: Un estudio empírico. Psicológica, 19,273-258. 


\section{Anexo}

\section{Material complementario.}

Inventario para la medición de la articulación entre la persona y la organización (versión validada en lengua española).

\section{Inventario APOE}

Los valores en la organización constituyen el elemento de la cultura que nos permite identificarnos o no con la misma. Las siguientes afirmaciones tienen como objetivo explorar la relación entre valores organizacionales y valores personales, por lo que le pedimos que marque su opinión al respecto, de acuerdo con las opciones disponibles:

0- Desacuerdo Total

1- Gran desacuerdo

2- Poco desacuerdo

3- Ni de acuerdo ni en desacuerdo

4- Concuerdo poco

5- Concuerdo mucho

6- Concuerdo totalmente

\begin{tabular}{|c|c|c|c|c|c|c|c|}
\hline Ítems & $\mathbf{0}$ & 1 & 2 & 3 & 4 & 5 & 6 \\
\hline $\begin{array}{l}\text { 1. Reconozco fácilmente los valores y la cultura de la } \\
\text { organización a la que pertenezco. }\end{array}$ & & & & & & & \\
\hline $\begin{array}{l}\text { 2. Me identifico con los valores y la cultura de la } \\
\text { organización a la que pertenezco.* }\end{array}$ & & & & & & & \\
\hline $\begin{array}{l}\text { 3. En la organización a la que pertenezco, considero que } \\
\text { los valores y la cultura están explícitos en el } \\
\text { comportamiento de las personas. }\end{array}$ & & & & & & & \\
\hline $\begin{array}{l}\text { 4. Mis valores personales están en conflicto con los } \\
\text { valores de mi trabajo / función. }\end{array}$ & & & & & & & \\
\hline $\begin{array}{l}\text { 5. En la organización a la que pertenezco, considero que } \\
\text { los valores y la cultura están explícitamente definidos. }\end{array}$ & & & & & & & \\
\hline $\begin{array}{l}\text { 6. Mis valores personales están en conflicto con los } \\
\text { valores de la organización a la que pertenezco. }\end{array}$ & & & & & & & \\
\hline $\begin{array}{l}\text { 7. Los valores y la cultura de la organización a la que } \\
\text { pertenezco son un reflejo de mis valores y mi cultura. }\end{array}$ & & & & & & & \\
\hline $\begin{array}{l}\text { 8. Las personas que pertenecen a mi organización saben } \\
\text { cuáles son los valores y la cultura de la organización. }\end{array}$ & & & & & & & \\
\hline $\begin{array}{l}\text { 9. Si los valores de la organización a la que pertenezco } \\
\text { fueran contrarios a los que existen actualmente, } \\
\text { difícilmente me identificaría con ellos. }\end{array}$ & & & & & & & \\
\hline $\begin{array}{l}\text { 10. Cuestiono mis valores personales cuando estoy } \\
\text { trabajando. }\end{array}$ & & & & & & & \\
\hline $\begin{array}{l}\text { 11. Las personas del área donde trabajo saben cuáles son } \\
\text { los valores y la cultura de la organización. }\end{array}$ & & & & & & & \\
\hline $\begin{array}{l}\text { 12. Las personas externas a la organización a la que } \\
\text { pertenezco la identifican por sus valores y su cultura. }\end{array}$ & & & & & & & \\
\hline
\end{tabular}


13. Los comportamientos que mi organización valor están en conflicto con mis valores personales.

14. Las cosas que yo valoro en mi vida son semejantes a las cosas que la organización a la que pertenezco valora.

15. Tengo que olvidar mis objetivos personales para poder cumplir con los objetivos de mi organización.

16. Mis valores personales coinciden con los valores y la cultura de la organización a la que pertenezco.

17. La razón por la que prefiero mi organización en comparación con otras son los valores que esta defiende y apoya.

18. Tengo que dejar de ser yo mismo para poderme adaptar a la organización a la que pertenezco.

19. Los objetivos de mi organización están en correspondencia con mis objetivos personales.

Nota: *indica que el ítem fue removido y no forma parte de la versión final del Inventario APO para Ecuador 